Supporting information for:

Structural basis of substrate range and enantioselectivity of two (S)-selective $\omega-$ transaminases

Niels van Oosterwijk ${ }^{\mathrm{a}}$, Simon Willies ${ }^{\mathrm{b}}$, Johan Hekelaar ${ }^{\mathrm{a}}$, Anke C. Terwisscha van Scheltinga ${ }^{\mathrm{a}}$, Nicholas J. Turner ${ }^{\mathrm{b}}$ and Bauke W. Dijkstra ${ }^{\mathrm{a} *}$

${ }^{a}$ Laboratory of Biophysical Chemistry, Groningen Biomolecular Sciences and Biotechnology Institute (GBB), University of Groningen, Nijenborgh 7, 9747 AG Groningen, The Netherlands

${ }^{b}$ School of Chemistry, Manchester Institute of Biotechnology, University of Manchester, 131 Princess Street, Manchester, M1 7DN, UK

*Corresponding author

This file includes: Figure S1: SAXS scattering curves and Guinier plots Figure S2. Interactions of the PLP co-factor Figure S3. Configurations around the $\mathrm{C} 7$ atom of the external aldimine intermediate 
A
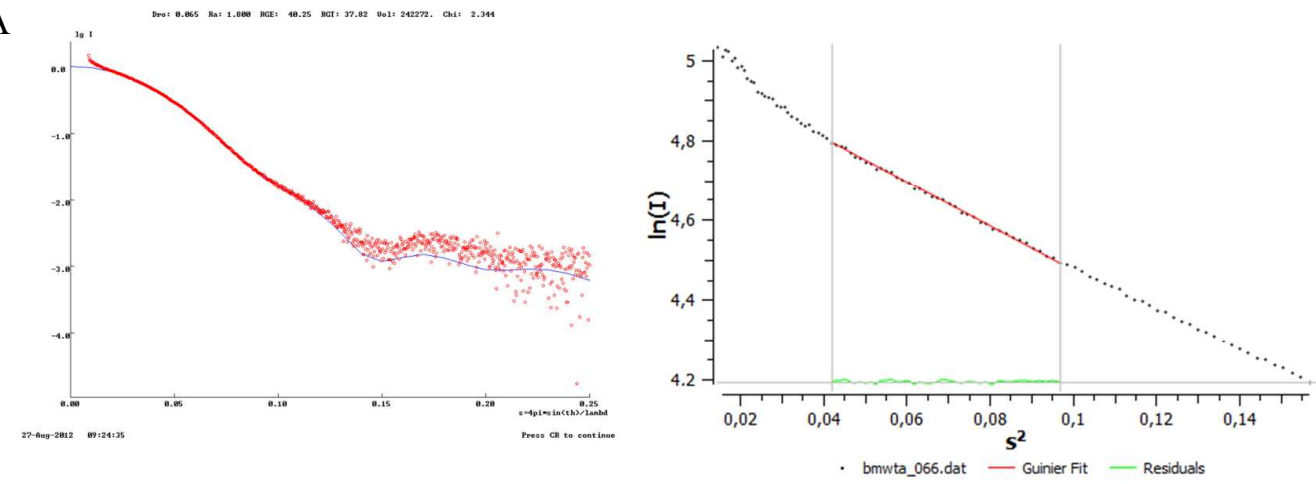

B
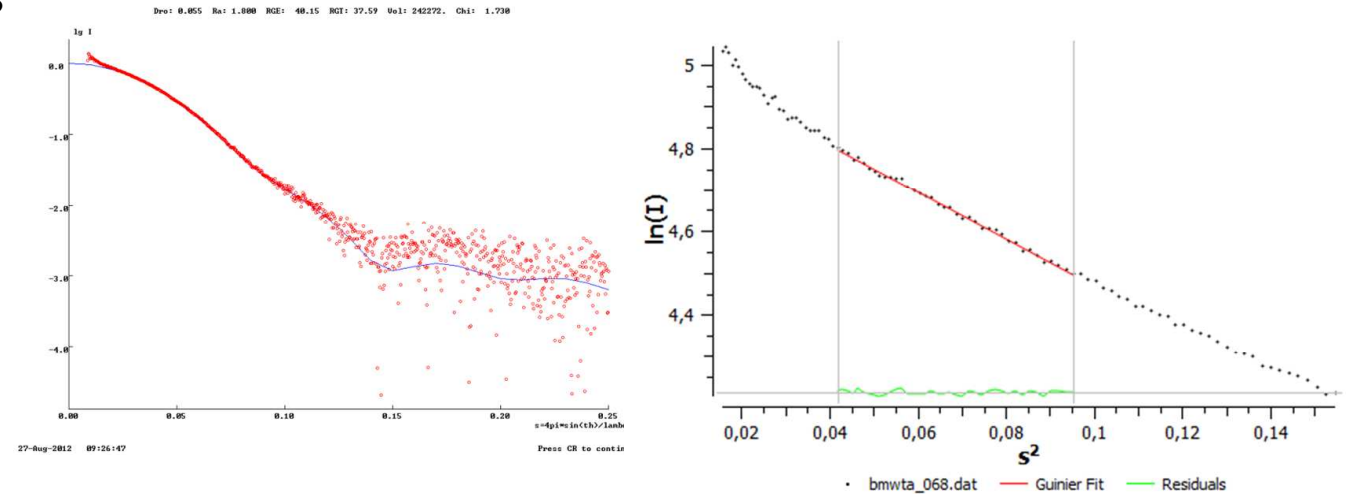

$\mathrm{C}$
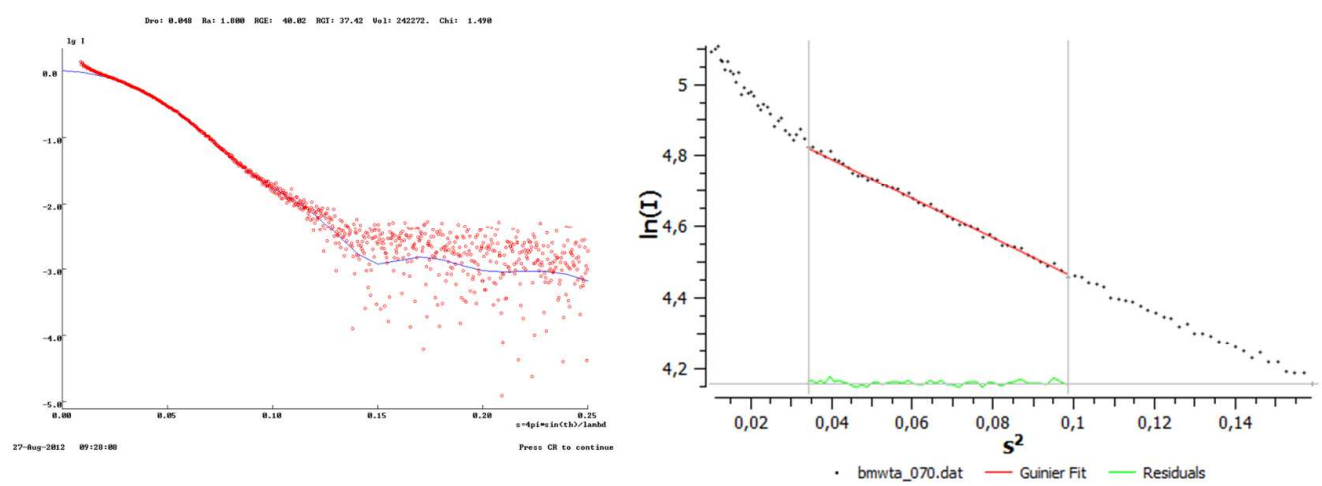

Figure S1

SAXS scattering curves and Guinier plots of BM- $\omega$ TA at protein concentrations of A) $5.45 \mathrm{mg} / \mathrm{ml}$, B) $2.61 \mathrm{mg} / \mathrm{ml}$, and C) $1.79 \mathrm{mg} / \mathrm{ml}$. The blue line shows the fit of the experimental SAXS scattering curves to the scattering curve calculated from the tetramer observed in the crystal structure. The $\mathrm{chi}^{2}$ values for the fits are $2.34,1.73$, and 1.49 for A), B), and C), respectively. 


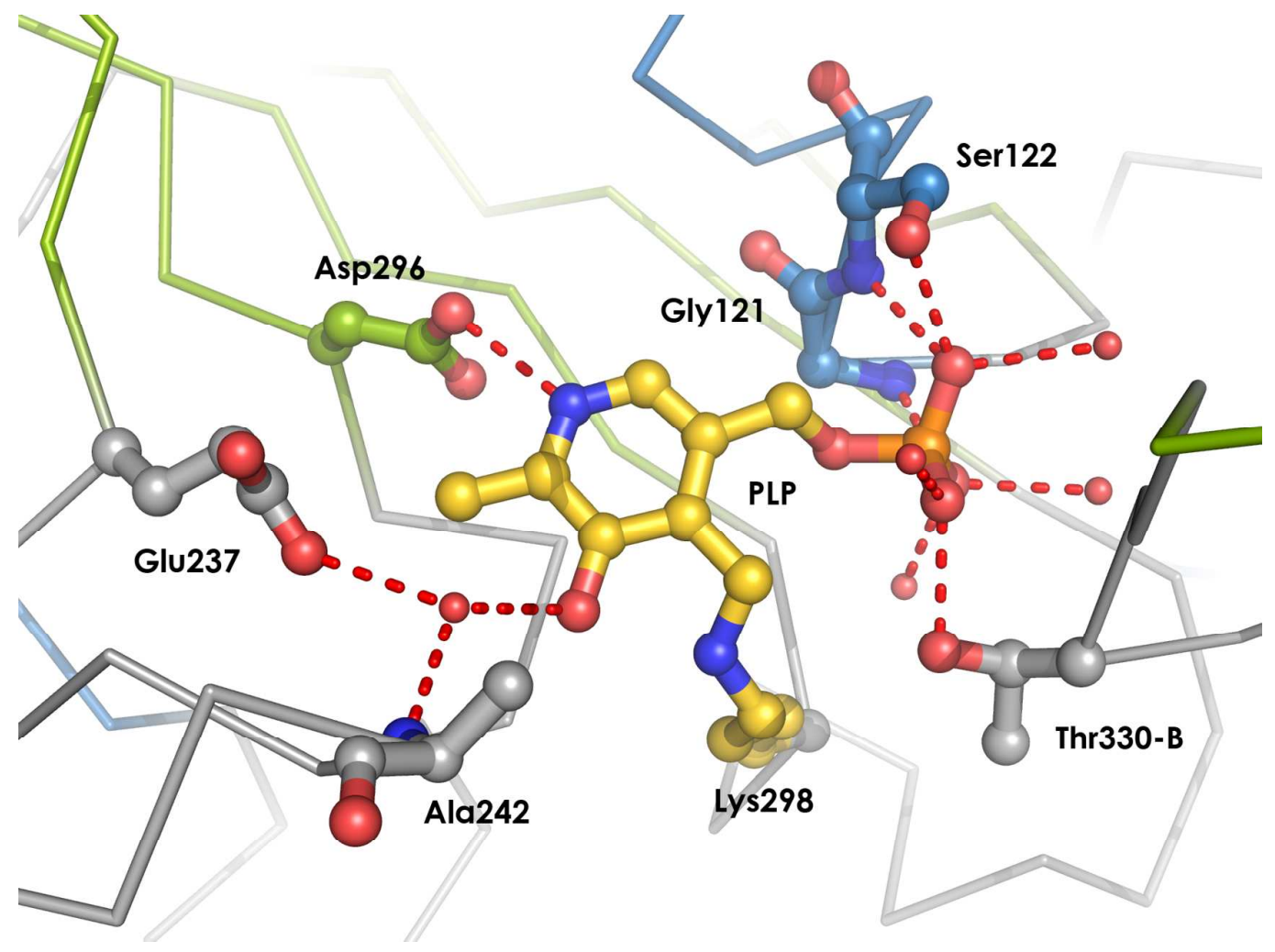

\section{Figure S2}

Interactions of the PLP co-factor in BM- $\omega$ TA. The $\mathrm{C} \alpha$ trace is coloured according to the secondary structure ( $\alpha$-helices in blue, $\beta$-strands in green, and loops in grey). The residues and water molecules involved in hydrogen bonding interactions with the PLP are indicated. 


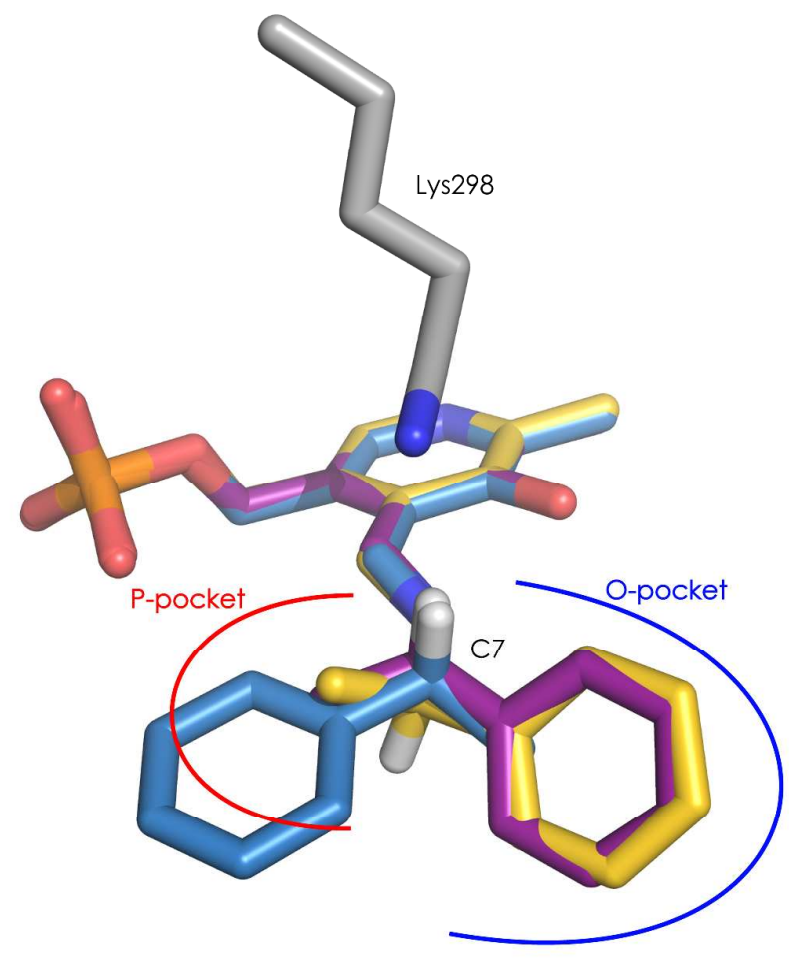

\section{Figure S3}

Configurations around the $\mathrm{C} 7$ atom of the external aldimine intermediate, as discussed in the main text. Schematic representations of the O- and P-pockets are shown. The experimentally determined (unproductive) binding mode of PLP-(R)- $\alpha-$ MBA is shown in yellow, with its $\mathrm{C} 7$ hydrogen atom pointing away from the catalytic Lys298. Inversion of the chiral centre of C7, while keeping the methyl and phenyl groups in the same pockets, would result in a productive binding mode of PLP- $(S)-\alpha-$ MBA (purple) with the C7-hydrogen pointing towards the catalytic Lys298. In blue, a putative productive binding mode of $(R)-\alpha-\mathrm{MBA}$ is shown, with its phenyl group in the P-pocket and its methyl group in the O-pocket, and the C7-hydrogen pointing towards the catalytic Lys298. However, inspection of the P-pocket in the X-ray structure shows that it is too small to accommodate the larger phenyl group. 